\title{
Mechanistic Insights in the Reversal of Enantioselectivity of Chiral Catalysts by Achiral Catalysts in Asymmetric Autocatalysis
}

\author{
François Lutz, ${ }^{\dagger}$ Takashi Igarashi, ${ }^{\dagger}$ Tomoyuki Kinoshita, ${ }^{\dagger}$ Mai Asahina, ${ }^{\dagger}$ Koichi \\ Tsukiyama, "Tsuneomi Kawasaki ${ }^{\dagger}$ and Kenso Soai* ${ }^{* \dagger}$ \\ Department of Applied Chemistry, and Department of Chemistry, Tokyo University of Science, \\ Kagurazaka, Shinjuku-ku, Tokyo, 162-8601 Japan
}

\section{Supporting Information}

Typical Experimental Procedure

Deconvolution Catalysis/Autocatalysis

Evolution of Yield versus Time for Asymmetric Autocatalysis Initiated by Mixtures of $(1 R, 2 S)$-DMNE 3 and DBAE 4

Representative Simulations of the Variation of Initial Rate with the Catalytic Mixture

Representative Simulations of the Variation of Initial Enantiomeric Excess of the Product with the Catalytic Mixture

Typical Experimental Procedure:

To a mixture of $(1 R, 2 S)$ - $N, N$-dimethylnorephedrine $3(5.0 \mathrm{~mL}$ of a $50 \mathrm{mM}$ toluene solution, 0.25 mmol) and achiral $N, N$-dibutylaminoethanol $4(5.0 \mathrm{~mL}$ of a $50 \mathrm{mM}$ toluene solution, $0.25 \mathrm{mmol})$ and anhydrous naphthalene $(2.0 \mathrm{~mL}$ of a $0.25 \mathrm{M}$ toluene solution, $0.50 \mathrm{mmol})$ was slowly added diisopropylzinc $(1.8 \mathrm{~mL}, 1 \mathrm{M}$ in toluene, $1.8 \mathrm{mmol})$ at $0{ }^{\circ} \mathrm{C}$. After stirring the mixture for $30 \mathrm{~min}$, a chilled toluene solution ( $3 \mathrm{~mL}$ ) of 2-tert-butylethynylpyrimidine-5-carbaldehyde 1 (94 mg, 0.50 mmol) was rapidly added. Aliquots $(0.5 \mathrm{~mL})$ were regularly (every $30 \mathrm{~s}$ ) taken via syringes and quenched with a mixture of aqueous ammonia and saturated aqueous ammonium chloride solution 
$(2 \mathrm{~mL}$ ). The organic layers were filtered and analyzed by HPLC (column Sumipax ODS A-05-601; eluent: methanol/water 7/3; flow rate: $0.9 \mathrm{~mL} / \mathrm{min}$; retention times: aldehyde 1: 6.5 min, alkanol 2: $10.2 \mathrm{~min}$, toluene: $14.7 \mathrm{~min}$, naphthalene: $19.8 \mathrm{~min}$ ). The yield of the reaction was deduced from the relative amounts of alkanol 2 and naphthalene. The samples were then purified by preparative chromatography on silica gel (eluent: hexane/ethyl acetate 2/1) and the enantiomeric excess of alkanol 2 measured by HPLC (column Chiralcel OD-H; eluent: hexane/isopropanol 95/5; flow rate: $1 \mathrm{~mL} / \mathrm{min}$; retention times: $(S)-3: 11.2 \mathrm{~min},(R)-3: 15.7 \mathrm{~min})$.

\section{Deconvolution Catalysis/Autocatalysis:}

A previous kinetic study showed the presence of an incubation period of about 10 minutes before asymmetric autocatalysis starts. Moreover, it was also shown that the non-catalyzed addition is a very slow process (see reference $3 \mathrm{a}$ ).

On the other hand, asymmetric autocatalysis initiated by $20 \mathrm{~mol} \%$ of $(1 R, 2 S)$-DMNE 3 at $0{ }^{\circ} \mathrm{C}$ exhibits the following kinetic profile (Figure S-1):

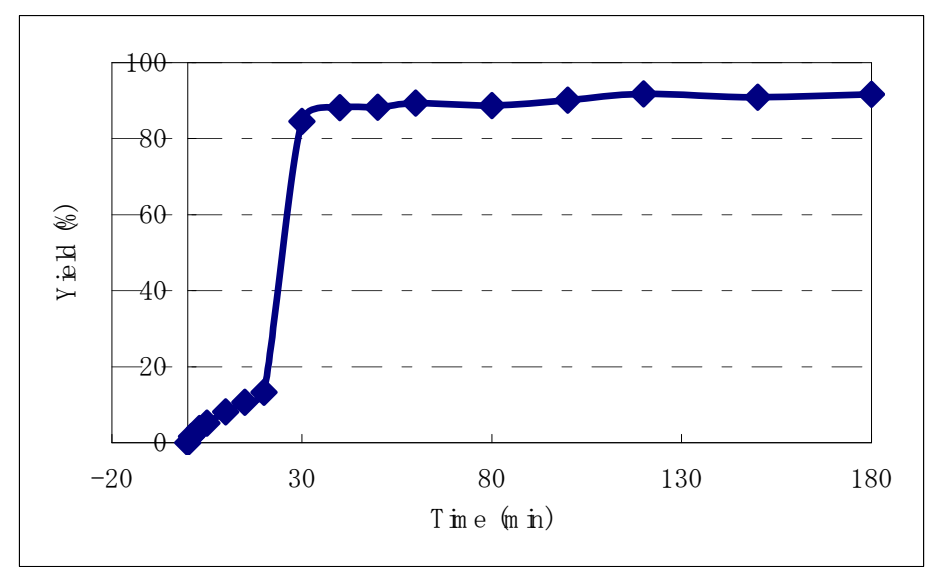

Figure S-1. Kinetic study of asymmetric autocatalysis initiated by $20 \mathrm{~mol} \%$ of $(1 R, 2 S)$-DMNE $\mathbf{3}$ at $0{ }^{\circ} \mathrm{C}$.

Similar kinetic profiles were obtained when asymmetric autocatalysis was initiated by mixtures of $(1 R, 2 S)$-DMNE 3 and DBAE 4 at $0{ }^{\circ} \mathrm{C}$, or DBAE alone. Thus, the beginning of the reaction (the first twenty minutes) corresponds to asymmetric catalysis, and the initial rate of the reaction corresponds to the catalytic activity of DMNE. This interpretation is also supported by the evolution of the ee of the alkanol versus time, which is constant until 20 minutes, then rapidly increases until 30 minutes (Figure S-2): 


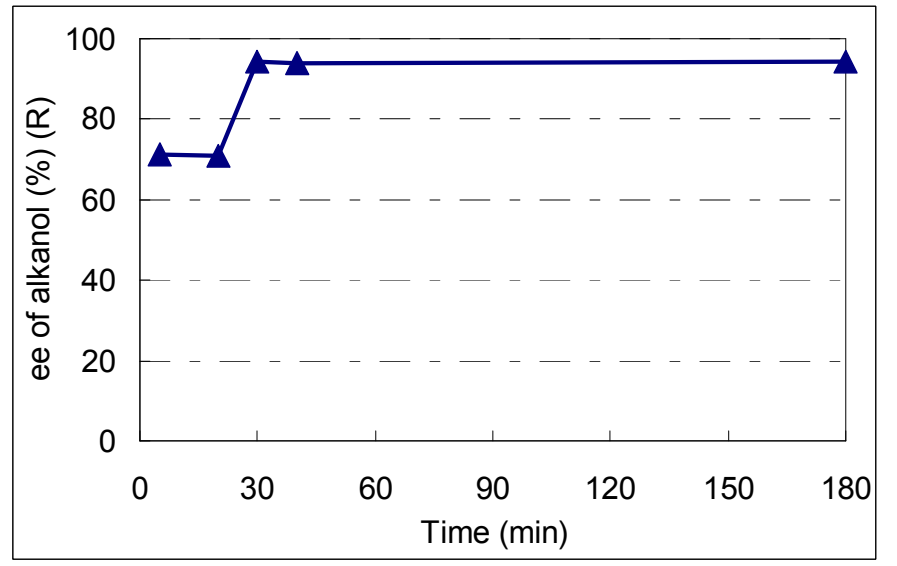

Figure S-2. Variation of the enantiomeric excess of alkanol with time in the asymmetric autocatalysis initiated by $20 \mathrm{~mol} \%$ of $(1 R, 2 S)$-DMNE 3 at $0{ }^{\circ} \mathrm{C}$.

\section{Evolution of Yield versus Time for Asymmetric Autocatalysis Initiated by Mixtures of $(1 R, 2 S)$-DMNE 3 and DBAE 4:}

The reaction was catalyzed by mixtures of $(1 R, 2 S)$-DMNE 3 or DBAE 4 (100 mol\% of catalysts in total), and the yield of the reaction was measured during the first five minutes (Figure S-3):

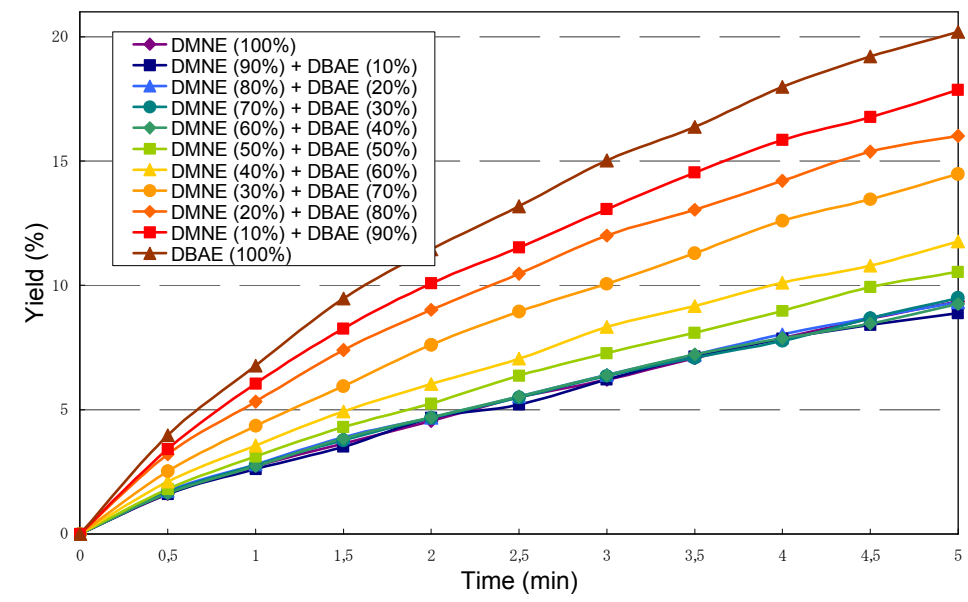

Figure S-3. Evolution of the yield with time with various mixtures of enantiopure DMNE or achiral DBAE.

The initial reaction rates were estimated by considering that the reaction follows a first order rate law for the aldehyde and diisopropylzinc (Equation 1):

$\frac{-d[1]}{d t}=k_{\text {cat }}[1]\left[i \operatorname{Pr}_{2} Z n\right]=k_{\text {cat }}[1]\left([1]+0.8 *[1]_{0}\right)$ (Equation 1)

After integration of Equation 1: 
$-\operatorname{Ln}\left[\frac{1.8 *(1-\text { Yield })}{1.8-\text { Yield }}\right]=0.8 *[1]_{0} * k_{\text {cat }} * t \quad$ (Equation 2$)$

The curves were simulated by a polynomial function, as shown on Figure S-4:

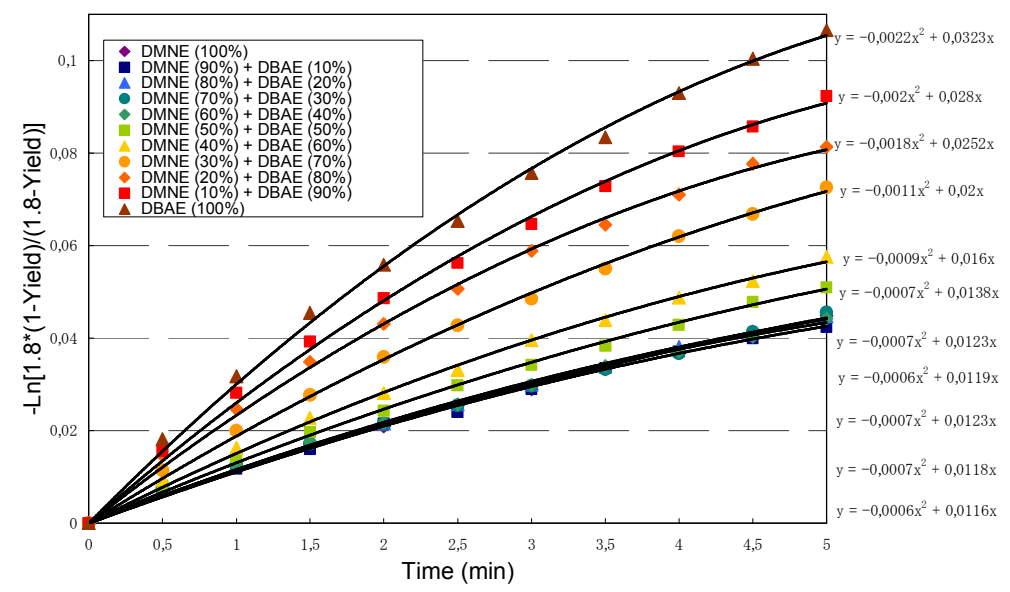

Figure S-4. Estimations of the initial rate of reaction.

Initial reaction rates $\mathrm{v}$ were then calculated from the initial slopes $\mathrm{s}_{0}$ of the polynomes (Equation 3 and Figure S-5):

$v=\frac{1.8}{0.8} * s_{0} *[1]_{0} \quad($ Equation 3$)$

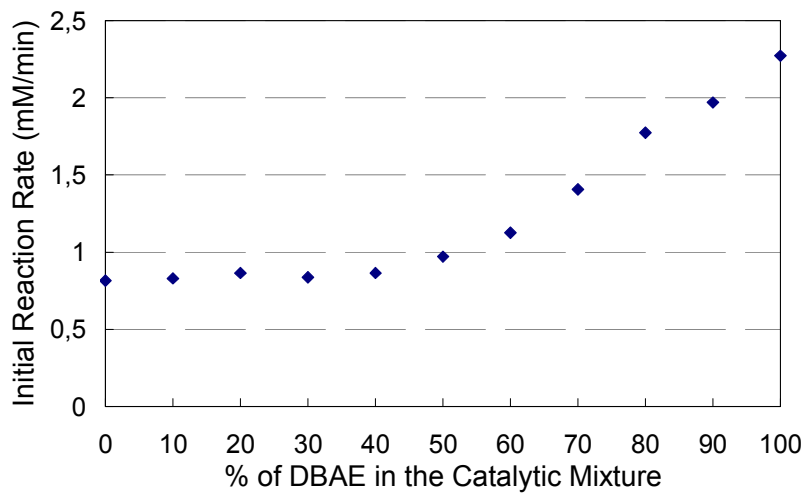

Figure S-5. Variation of the initial rate of reaction with the composition of the catalytic mixture.

It was then normalized (reference: rate of the reaction catalyzed by $100 \mathrm{~mol} \%$ of DMNE) to get Figure 1. 


\section{Determination of the Reaction Order in [Catalyst]:}

The reaction was catalyzed by 20,40 or $100 \mathrm{~mol} \%$ of $(1 R, 2 S)$-DMNE 3 or DBAE 4 , and the amount of diisopropylzinc was adjusted so that 1.8 equivalents with respect to the aldehyde remains after deprotonation of the ligand. The yield of the reaction was measured during the first five minutes, every thirty seconds (Figure S-6):

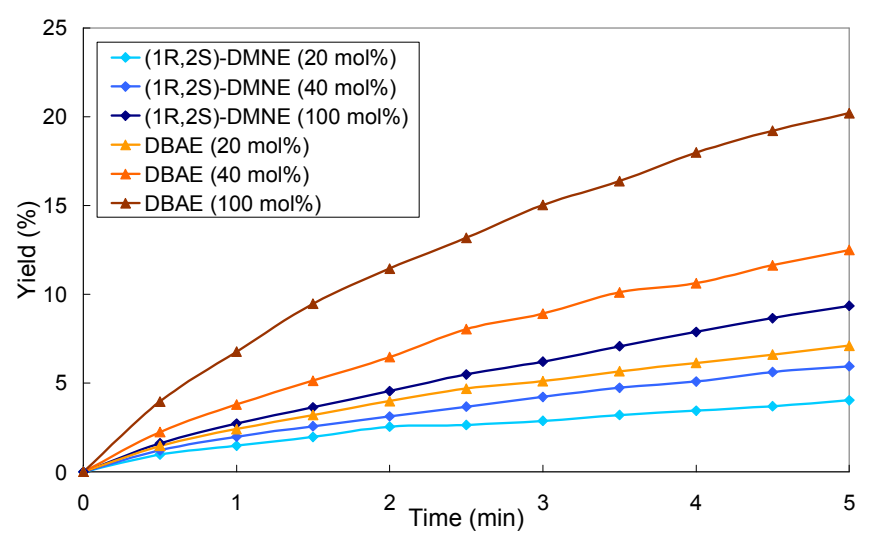

Figure S-6. Evolution of the yield with time with various amounts of enantiopure DMNE or achiral DBAE as catalyst.

The initial rate of reaction was then estimated by the precedent method, by considering a first order rate law for the aldehyde and diisopropylzinc (Equation 1). The reaction rate was shown to increase linearly with the amount of catalyst, so the reaction was considered to be first order with respect to the catalyst concentration (Equation 4)

\section{$k_{\text {cat }}=k *[C a t] \quad($ Equation 4)}

In the presence of the two catalysts, this gives rise to Equation 5:

$v=a v_{a}+b v_{b}+c v_{c}$ (Equation 5) 
Representative Simulations of the Variation of Initial Rate with the Catalytic Mixture:

$\mathrm{K}=\mathbf{0}$ (no heteroassociation):

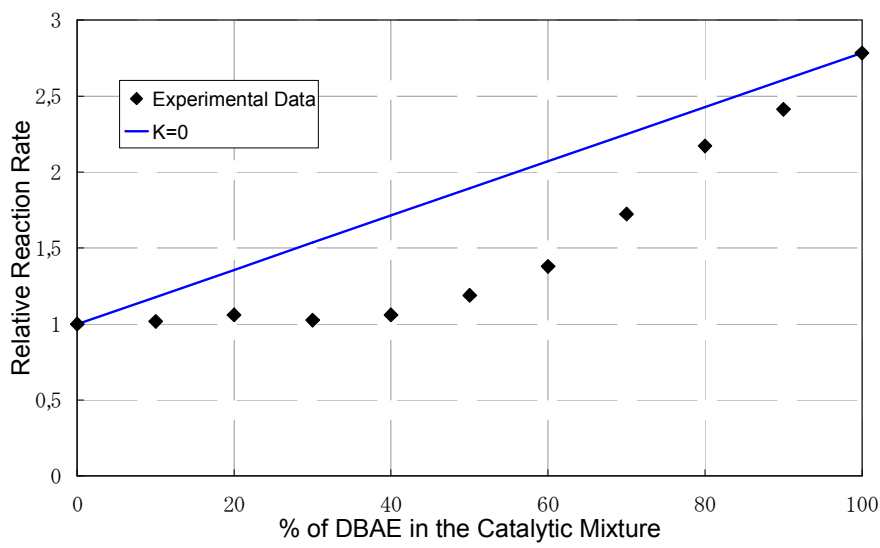

$\mathrm{K}=4$ (statistical distribution):

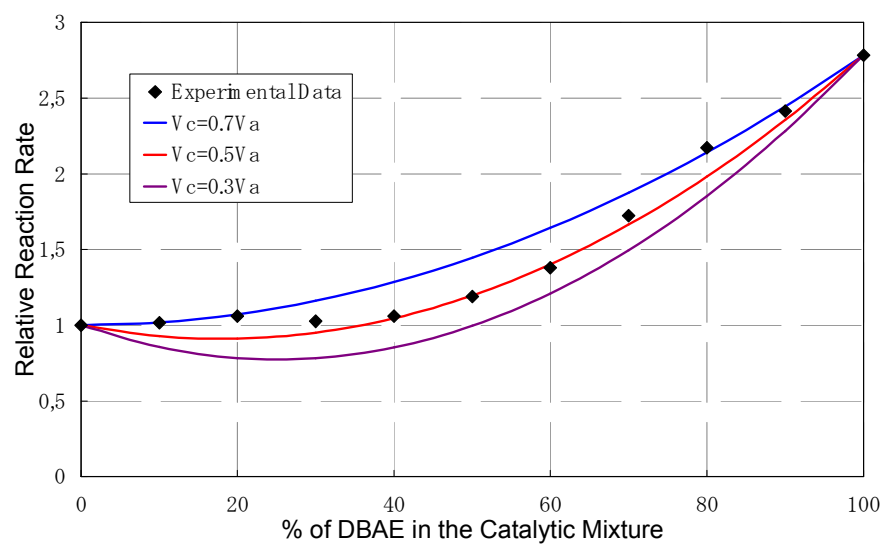

$\mathrm{K}=10$ :

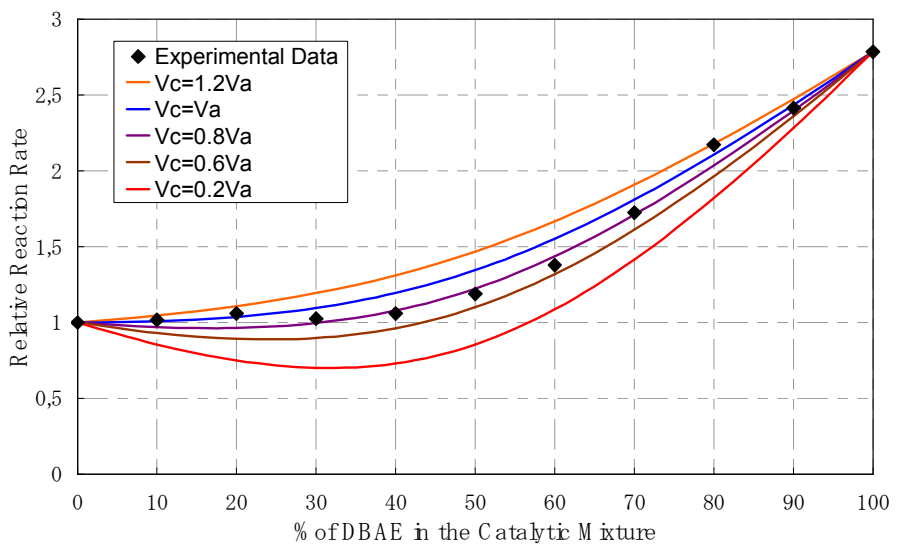


S-7 -

$K=\mathbf{2 5}$ :

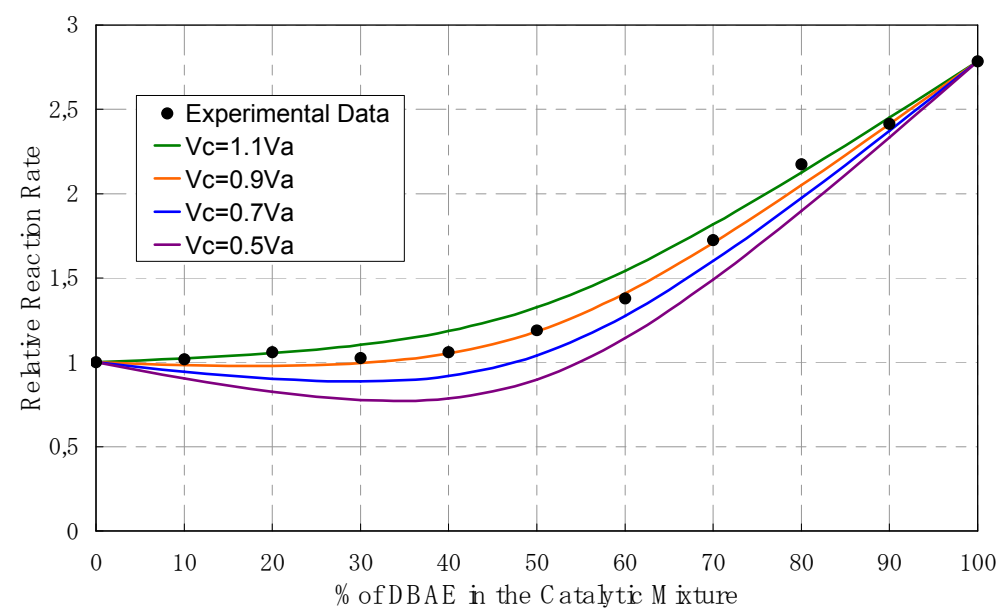

$K=100$ :

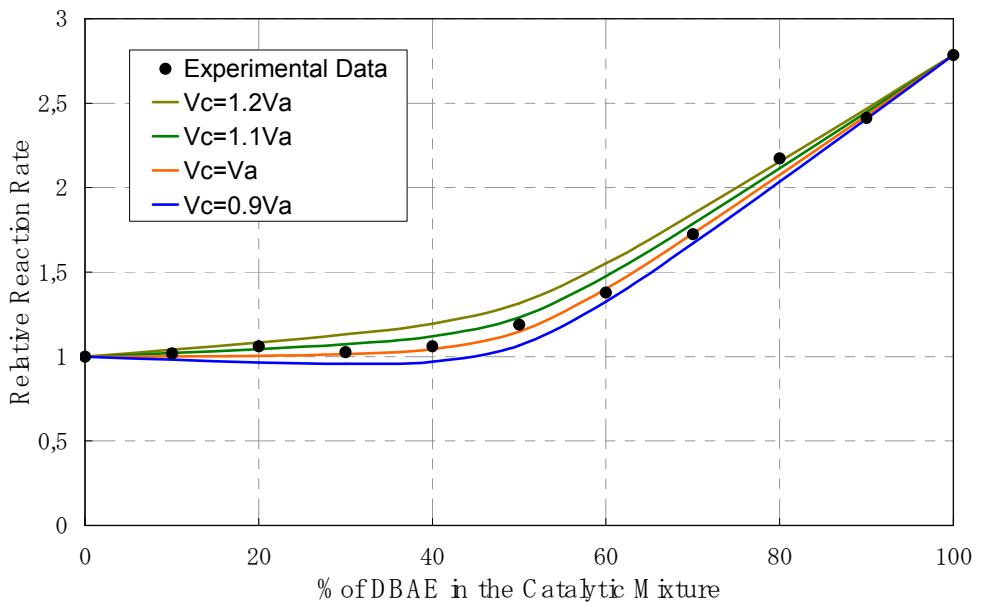

$K=1000$ :

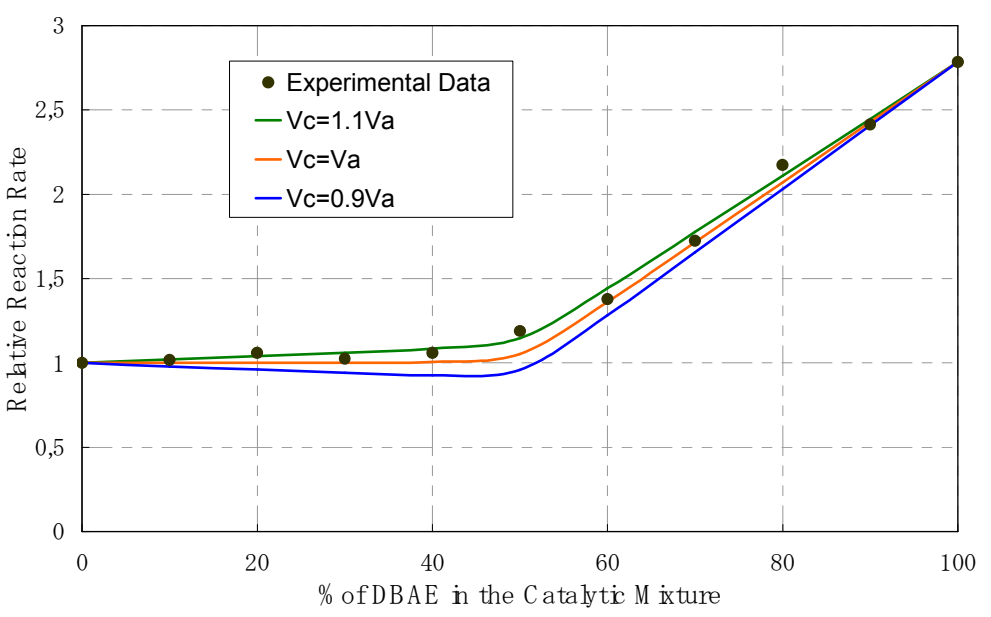


$\mathbf{K} \rightarrow \infty$ (complete heteroassociation) :

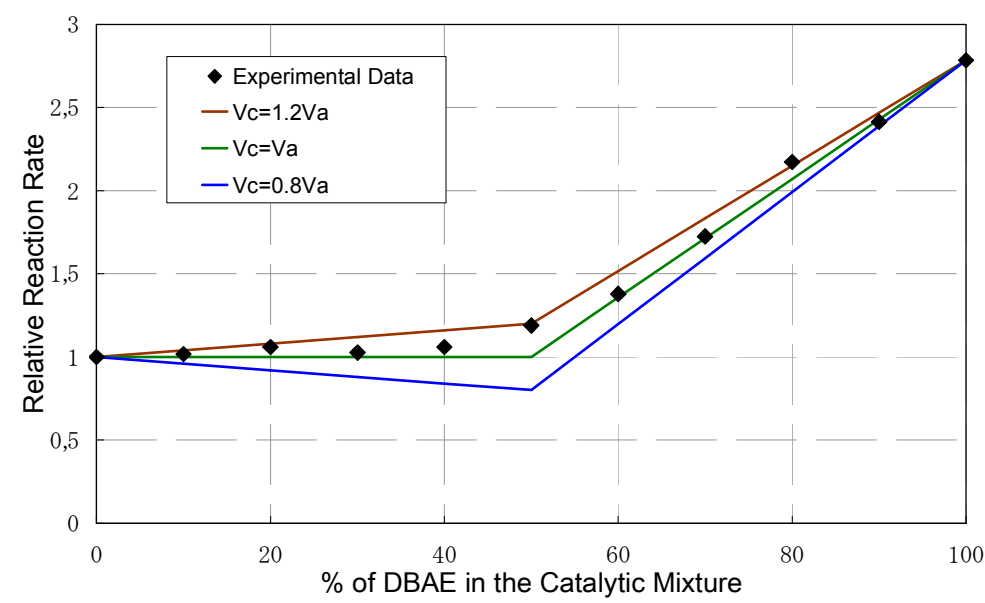


Representative Simulations of the Variation of Initial Enantiomeric Excess of the Product with the Catalytic Mixture:

$\mathrm{K}=\mathbf{0}$ (no heteroassociation):

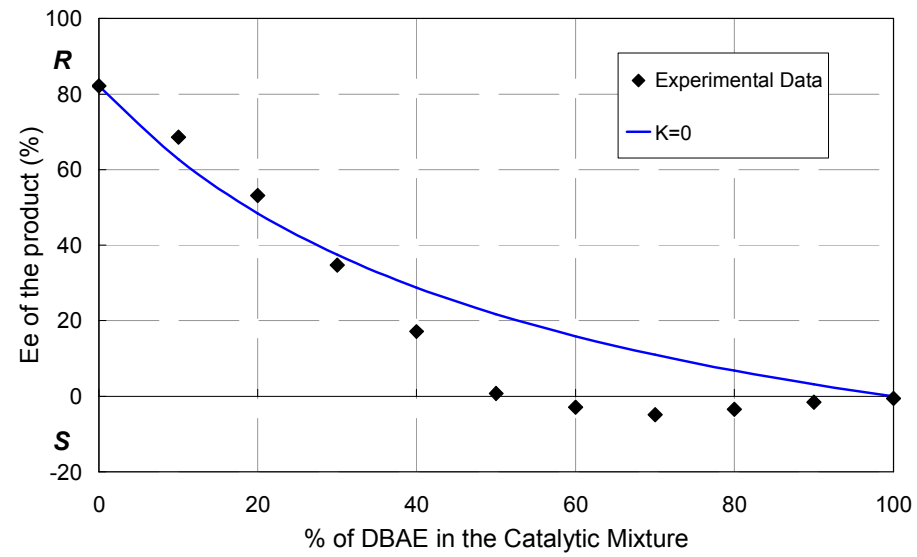

$K=4$ (statistical distribution):

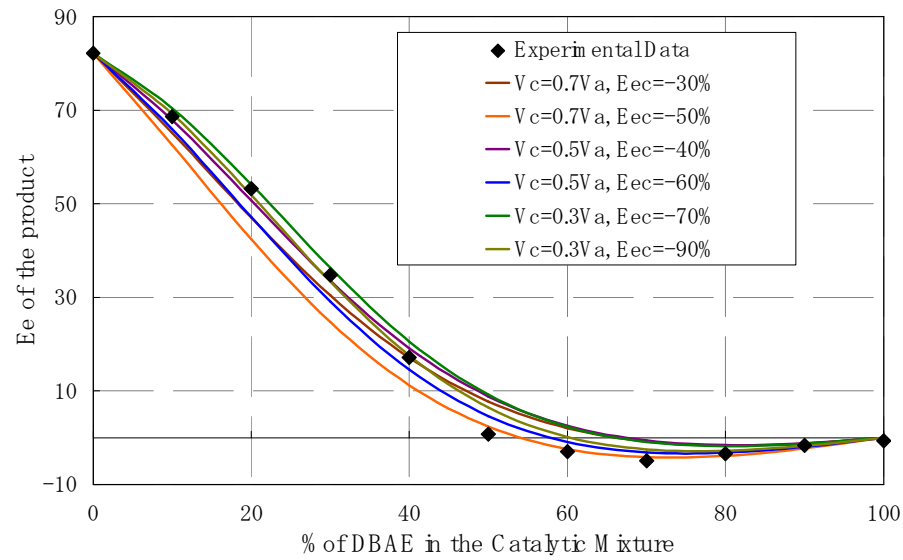

$\mathrm{K}=10$ :

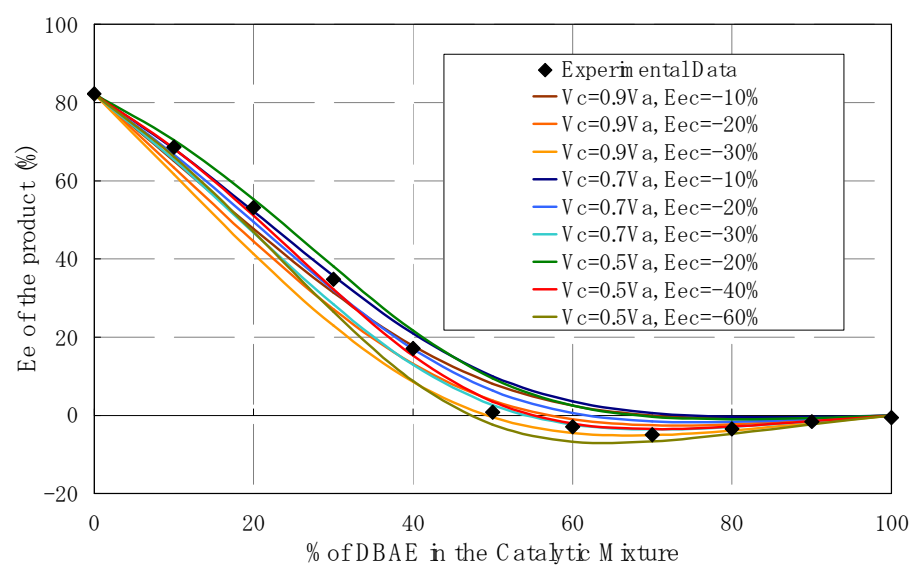


$K=25$ :

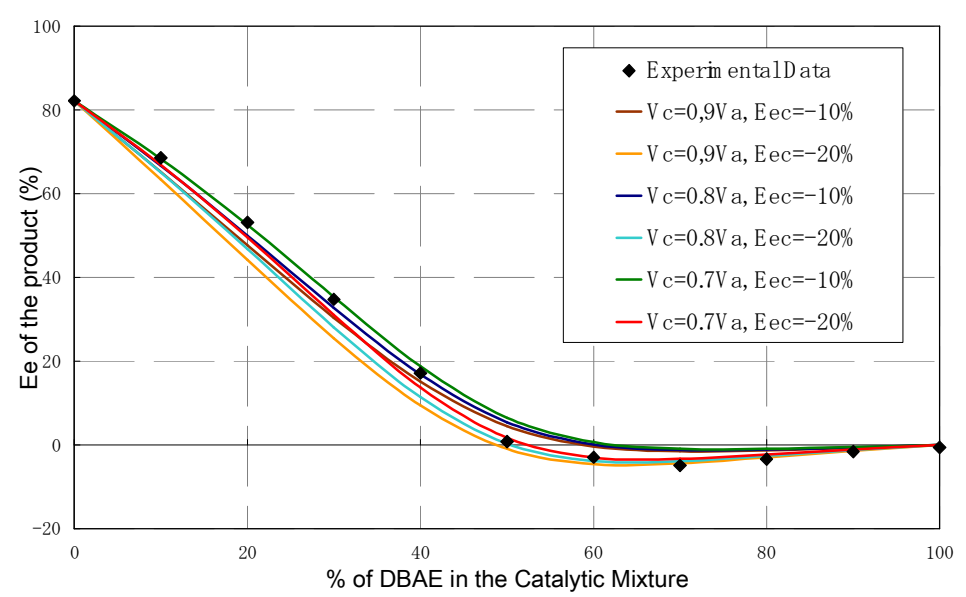

$K=100$ :

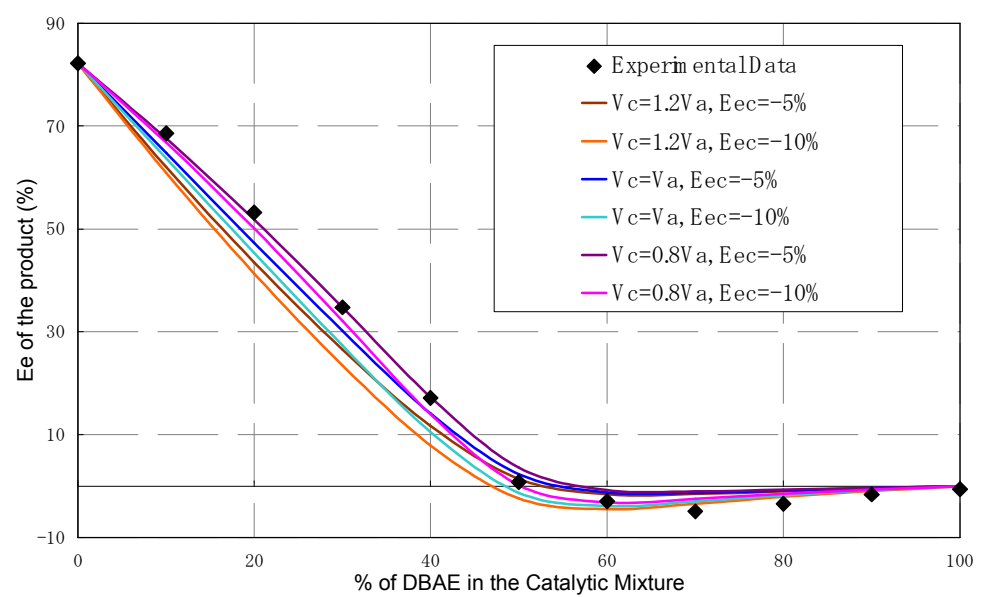

$K=1000$ :

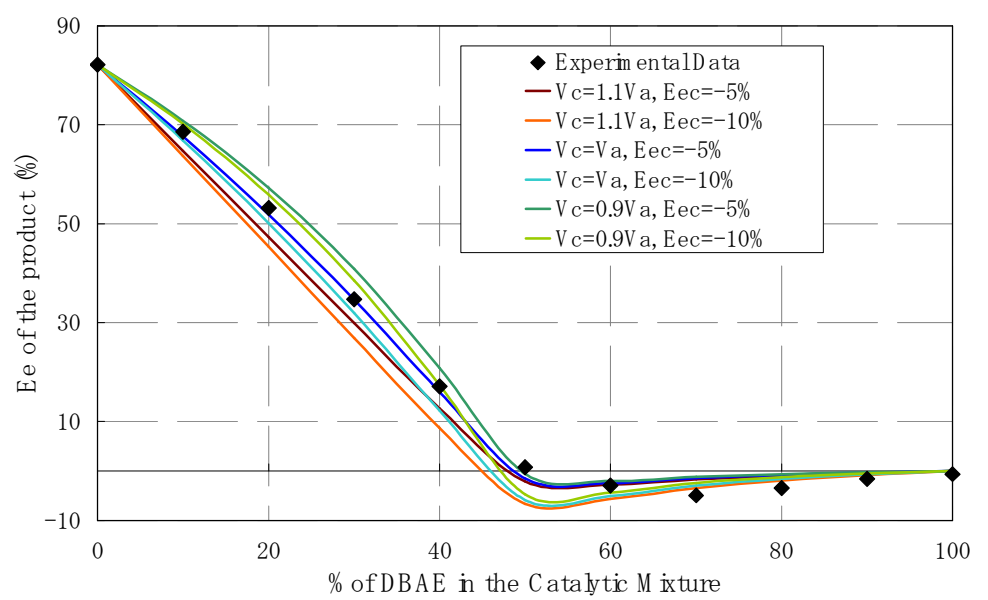


$\mathbf{K} \rightarrow \infty$ (complete heteroassociation):
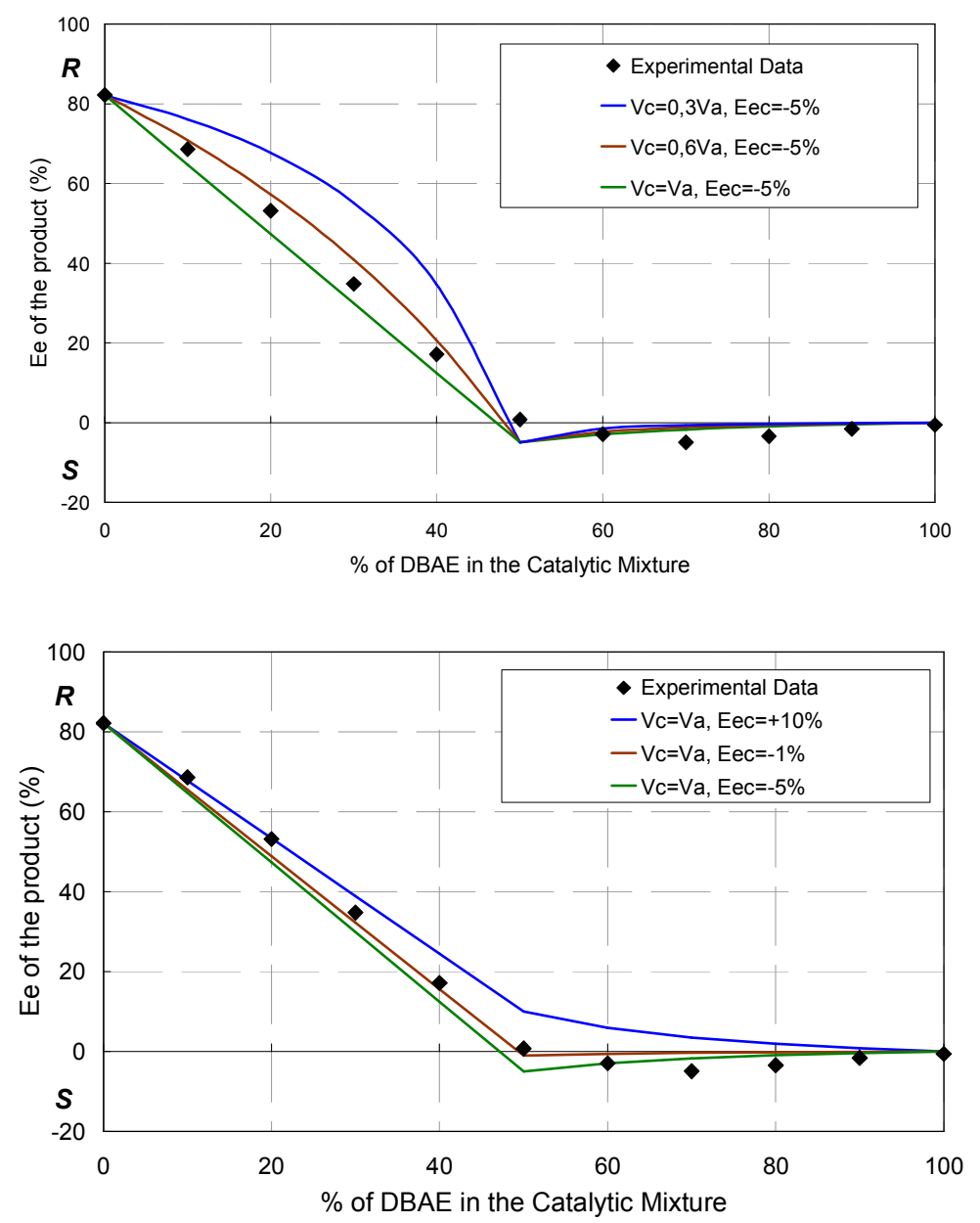

\section{NMR experiments}

All the following experiments were carried out in deuterated toluene $\left(\mathrm{C}_{7} \mathrm{D}_{8}\right)$, freshly distillated from sodium/benzophenone. Solution of diisopropylzinc $\left(i-\mathrm{Pr}_{2} \mathrm{Zn}\right)$ in $\mathrm{C}_{7} \mathrm{D}_{8}(0.1 \mathrm{~mol} / \mathrm{L})$ was bought from Tri Chemical.

A typical experimental procedure is as follows: in a two-neck flask was added the amino alcohol $(0.125 \mathrm{mmol})$, solved in $0.25 \mathrm{~mL}$ of $\mathrm{C}_{7} \mathrm{D}_{8}$ under argon. Diisopropylzinc $(0.375 \mathrm{mmol}, 3.75 \mathrm{~mL}$ of a $0.1 \mathrm{M}$ solution in $\mathrm{C}_{7} \mathrm{D}_{8}, 3$ equiv.) was slowly added at room temperature and stirred for 30 minutes. The flask was transferred in a glove box, itself put under vacuum then filled with argon. In the glove box an aliquot of the solution was transferred in an NMR sample tube, which was closed with Parafilm. NMR experiments were then taken on a Bruker AV-600 MHz at room temperature. The calibration was performed with the methyl group of $\mathrm{C}_{7} \mathrm{D}_{8}$ centered at $2.09 \mathrm{ppm}$. All the samples were obtained as clear solutions, in the flask as well as in the NMR sample tube. 
In these samples, the concentrations in amino alcohol $(31.2 \mathrm{mM})$ and diisopropylzinc $(62.5 \mathrm{mM}$ after deprotonation of the amino alcohol) are the same than in the experiments described in our manuscript. The aldehyde, however, is absent, because it would react with $i-\mathrm{Pr}_{2} \mathrm{Zn}$. Moreover, the temperature is different (room temperature instead of $0{ }^{\circ} \mathrm{C}$ ).

Sample Lutz 1947 : $(1 R, 2 S)-N, N$-Dimethylnorephedrine (DMNE) in $\mathrm{C}_{7} \mathrm{D}_{8}(31.2 \mathrm{mM})$.

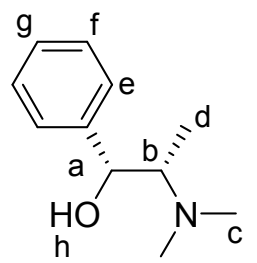

$0.69\left(\mathrm{~d}, J=6.6 \mathrm{~Hz}, 3 \mathrm{H}, \mathrm{H}_{\mathrm{d}}\right) ; 2.04\left(\mathrm{~s}, 6 \mathrm{H}, \mathrm{H}_{\mathrm{c}}\right) ; 2.21\left(\mathrm{qd}, J_{l}=6.6 \mathrm{~Hz}, J_{2}=4.2 \mathrm{~Hz}, 1 \mathrm{H}, \mathrm{H}_{\mathrm{b}}\right) ; 3.19(\mathrm{~s}, 1 \mathrm{H}$, $\left.\mathrm{H}_{\mathrm{h}}\right) ; 4.80\left(\mathrm{~d}, J=4.2 \mathrm{~Hz}, 1 \mathrm{H}, \mathrm{H}_{\mathrm{a}}\right) ; 7.07-7.10\left(\mathrm{~m}, 1 \mathrm{H}, \mathrm{H}_{\mathrm{g}}\right) ; 7.17-7.21\left(\mathrm{~m}, 2 \mathrm{H}, \mathrm{H}_{\mathrm{f}}\right) ; 7.31-7.34(\mathrm{~m}, 2 \mathrm{H}$, $\left.\mathrm{H}_{\mathrm{e}}\right)$.

Sample Lutz 1948 : $N, N$-Dibutylaminoethanol (DBAE) in $\mathrm{C}_{7} \mathrm{D}_{8}(31.2 \mathrm{mM})$.

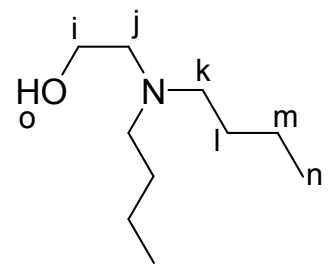

$0.86\left(\mathrm{t}, J=7.2 \mathrm{~Hz}, 6 \mathrm{H}, \mathrm{H}_{\mathrm{n}}\right) ; 1.17-1.24\left(\mathrm{~m}, 4 \mathrm{H}, \mathrm{H}_{\mathrm{m}}\right) ; 1.25-1.31\left(\mathrm{~m}, 4 \mathrm{H}, \mathrm{H}_{\mathrm{l}}\right) ; 2.23(\mathrm{t}, J=7.2 \mathrm{~Hz}, 4 \mathrm{H}$, $\left.\mathrm{H}_{\mathrm{k}}\right) ; 2.30\left(\mathrm{t}, J=5.4 \mathrm{~Hz}, 2 \mathrm{H}, \mathrm{H}_{\mathrm{j}}\right) ; 2.68\left(\mathrm{~s}, 1 \mathrm{H}, \mathrm{H}_{\mathrm{o}}\right) ; 3.44\left(\mathrm{t}, J=5.4 \mathrm{~Hz}, 2 \mathrm{H}, \mathrm{H}_{\mathrm{i}}\right)$.

Sample Lutz $1954: i$ - $\mathrm{Pr}_{2} \mathrm{Zn}$ in $\mathrm{C}_{7} \mathrm{D}_{8}(0.1 \mathrm{M})$.

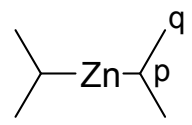

0.61 (septet, $\left.J=7.8 \mathrm{~Hz}, 2 \mathrm{H}, \mathrm{H}_{\mathrm{p}}\right) ; 1.20\left(\mathrm{~d}, J=7.8 \mathrm{~Hz}, 12 \mathrm{H}, \mathrm{H}_{\mathrm{q}}\right)$.

Sample Lutz 1950 : Isopropylzinc alkoxide of (1R,2S)-DMNE in $\mathrm{C}_{7} \mathrm{D}_{8}(31.2 \mathrm{mM})$.

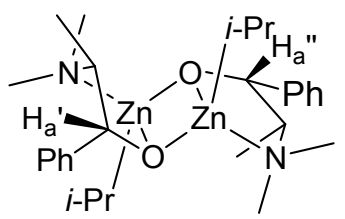

Complex spectrum. The presence of several peaks for each position can be interpreted as a result of aggregation (for example, in the dimeric form, protons $\mathrm{H}_{\mathrm{a}}$ ' and $\mathrm{H}_{\mathrm{a}}$ " are not equivalent). It can also be interpreted as a mixture of oligomers (monomer, dimers, tetramers, etc...) and/or 
conformers (endo/endo/trans, endo/exo/trans, exo/endo/trans, endo/endo/cis, etc...).

Signals corresponding to the alkoxide methine protons $\mathrm{H}_{\mathrm{a}}$ ' and $\mathrm{H}_{\mathrm{a}}$ ”: $5.17(\mathrm{~s}) ; 5.14(\mathrm{~s}) ; 5.10(\mathrm{~d}$, $J=3.6 \mathrm{~Hz}) ; 5.03(\mathrm{~s}), 4.91(\mathrm{~s})$.

Conclusion : isopropylzinc alkoxide of $(1 R, 2 S)$-DMNE is not only present as a simple monomeric form. The complexity of the spectrum can be allotted to equilibria between oligomers and/or equilibria between conformers.

Sample Lutz 1951 : Isopropylzinc alkoxide of DBAE (31.2 mM) in $\mathrm{C}_{7} \mathrm{D}_{8}$.

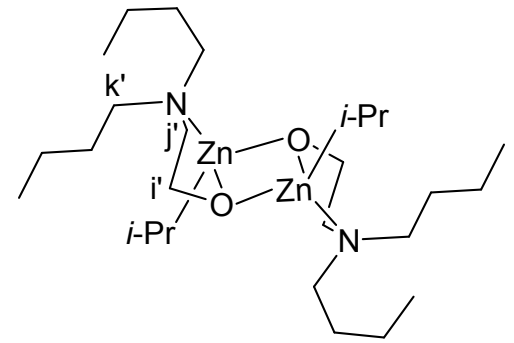

Two main "products" (oligomers or conformers) in a 2:1 ratio can be seen:

Signals corresponding to the protons $\mathrm{H}_{\mathrm{i}}$ ': major: $3.92(\mathrm{t}, J=5.1 \mathrm{~Hz})$; minor: $3.87(\mathrm{t}, J=5.1 \mathrm{~Hz})$.

Signals corresponding to the protons $\mathrm{H}_{\mathrm{j}}$ ': major: 2.55 (t, $J=4.8 \mathrm{~Hz}$ ); minor: 2.49 (t, $J=5.1 \mathrm{~Hz}$ ).

Signals corresponding to the protons $\mathrm{H}_{\mathrm{k}}{ }^{\prime}$ : major: $2.73(\mathrm{~m})$; minor: 2.63 (broad s).

Conclusion : the NMR spectrum of isopropylzinc alkoxide of DBAE shows the presence of an equilibrium between 2 oligomers or 2 conformers.

Sample Lutz 1953 : Isopropylzinc alkoxide of DBAE (15.6 mM) and (1R,2S)-DMNE (15.6 mM) in $\mathrm{C}_{7} \mathrm{D}_{8}$.

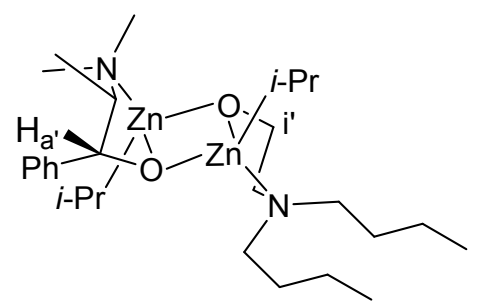

Complex spectrum. The presence of several peaks for each position can be interpreted as a mixture of oligomers and/or conformers.

Signals corresponding to the alkoxide methine protons $\mathrm{H}_{\mathrm{a}}{ }^{\prime}: 5.27$ (s); $5.02(\mathrm{~s}) ; 4.94$ (d, $\left.J=4.2 \mathrm{~Hz}\right)$. Signals corresponding to the protons $\mathrm{H}_{\mathrm{i}}{ }^{\prime}: 4.23(\mathrm{~m}) ; 3.97(\mathrm{~m}) ; 3.87(\mathrm{~m})$. 


\section{Comparisons with Lutz 1950 and Lutz 1951 :}

Signals corresponding to the alkoxide methine protons $\mathrm{H}_{2}$ ':

- almost complete disappearance of the major signals corresponding to the DMNE homoaggregate : 5.17 (s); $5.14(\mathrm{~s}) ; 5.10(\mathrm{~d}, J=3.6 \mathrm{~Hz}) ; 4.91$ (s).

- new major signals showing an interaction with DBAE : 5.27 (s); 4.94 (d, $J=4.2 \mathrm{~Hz}$ ).

- signal that may belong to the same species : 5.02 (s, Lutz 1953) and 5.03 (s, Lutz 1950).

Signals corresponding to the protons $\mathrm{H}_{\mathrm{i}}$ :

- complete disappearance of the major signal corresponding to the DBAE homoaggregate : $3.92(\mathrm{t}, J=5.1 \mathrm{~Hz})$.

- new major signals showing an interaction with DMNE : $4.23(\mathrm{~m}) ; 3.97(\mathrm{~m}) ; 3.87(\mathrm{~m})$.

- signal that may belong to the same species : 3.87 (t, $J=5.1 \mathrm{~Hz}$, Lutz 1951) and 3.87 (m, Lutz 1953).

\section{Conclusion :}

Our NMR experiments do not allow to affirm nor to cancel that the species are mainly dimeric. However, it shows that they are not simply monomeric.

In addition, they bring a strong additional support for the presence of interactions between the two catalysts: when they are mixed, the absence of the main signals corresponding to the homoaggregates and the presence of different signals corresponding to a mixed aggregate were observed. 
S-15 -
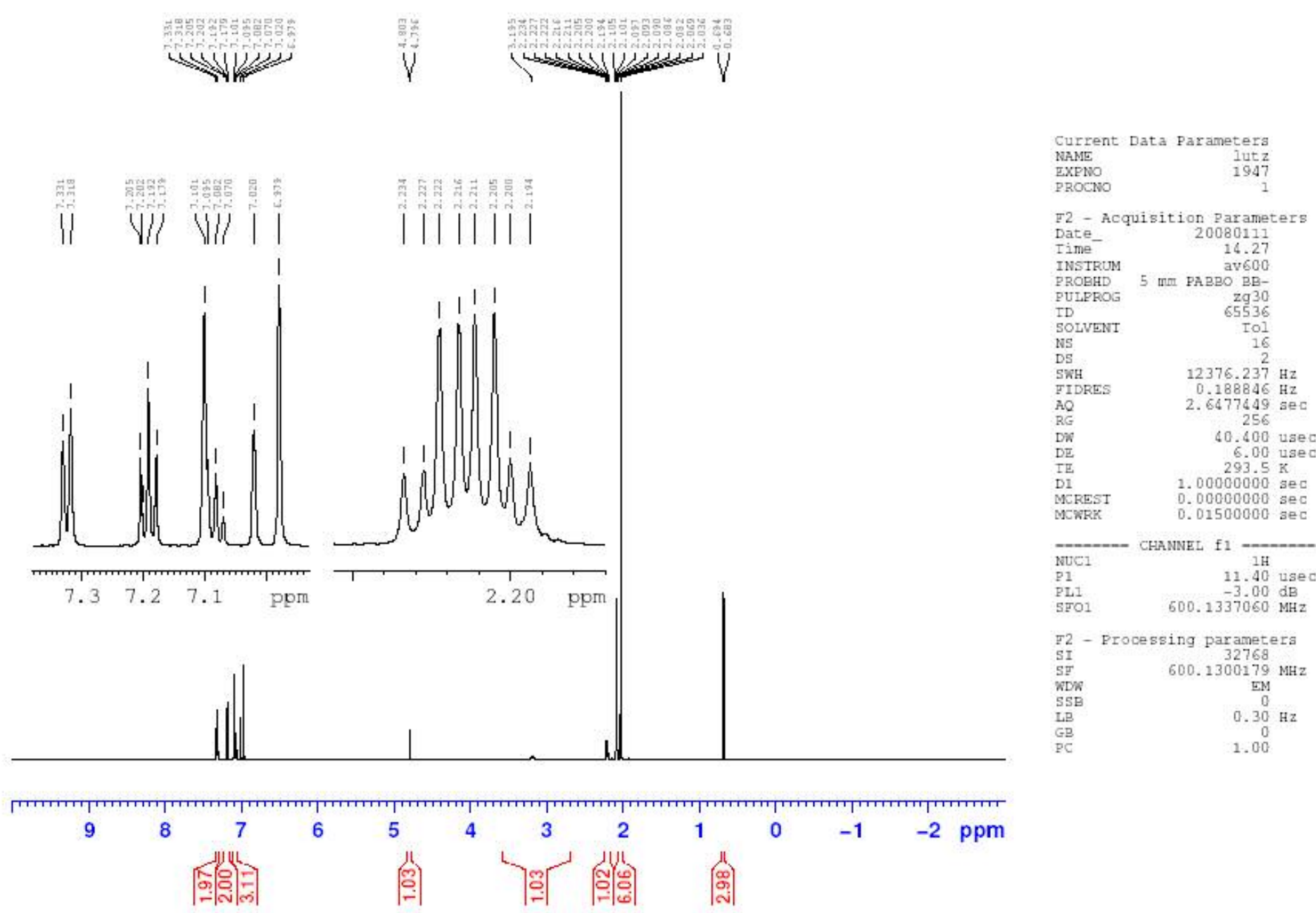


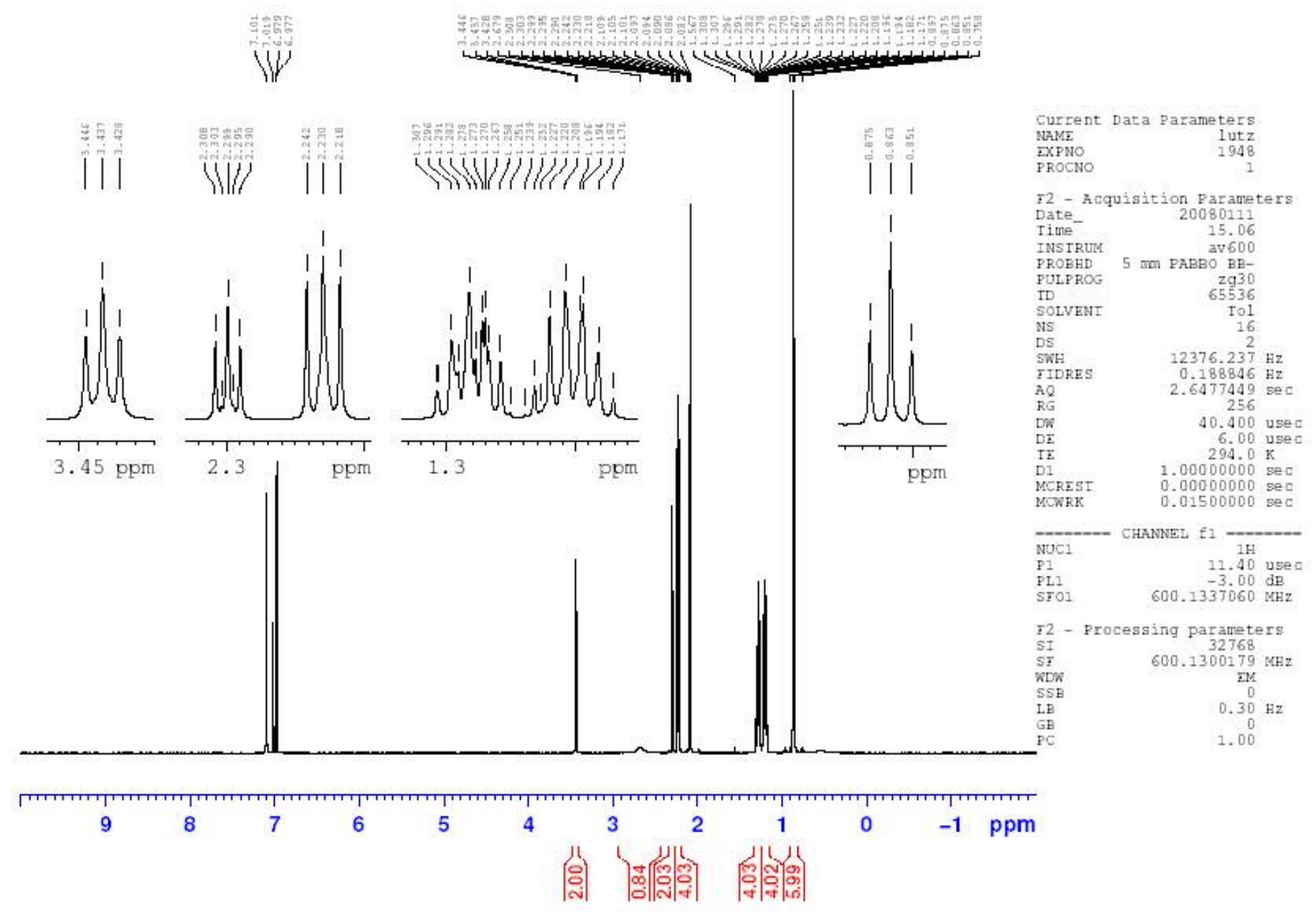


S-17 -

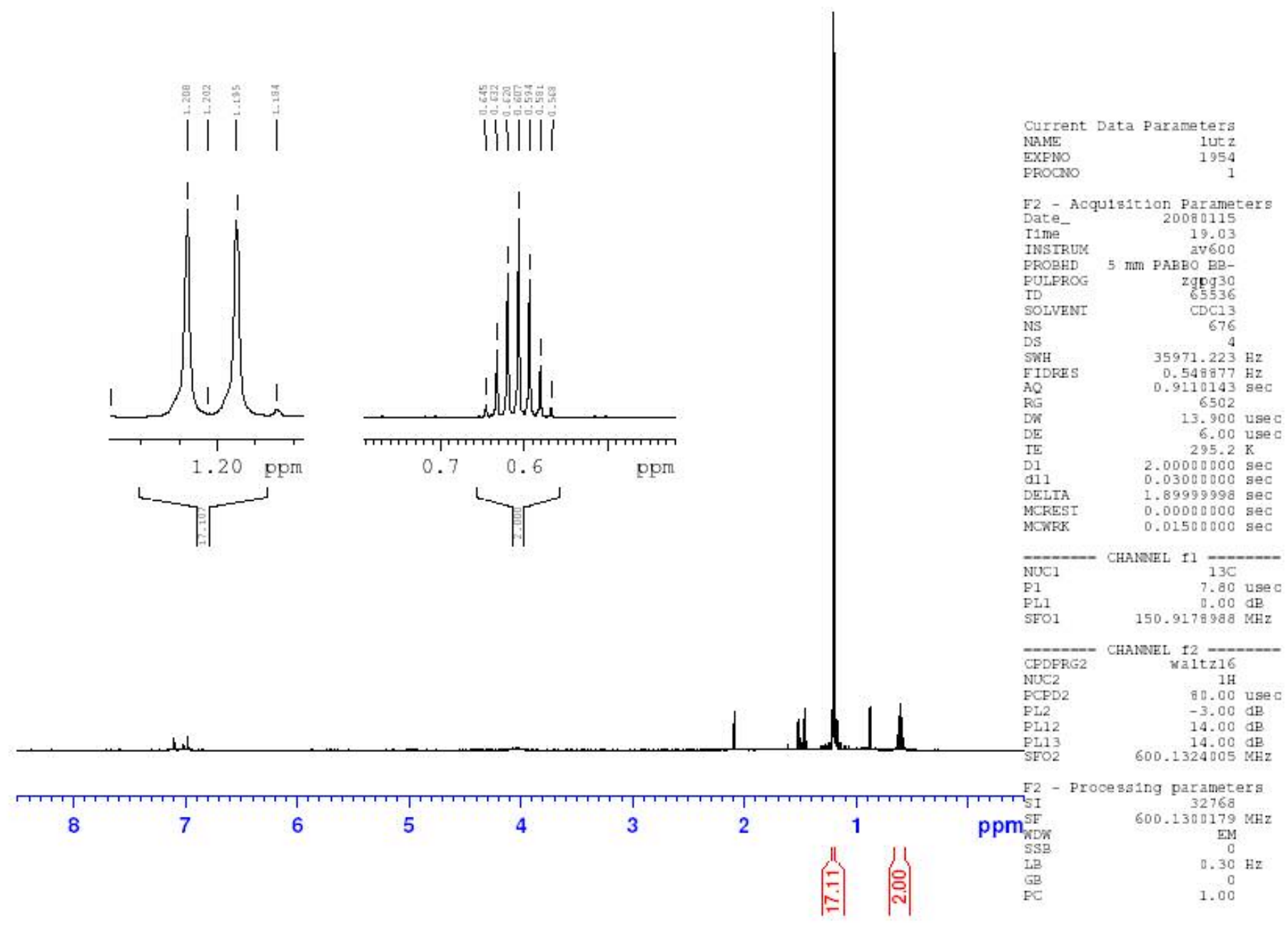



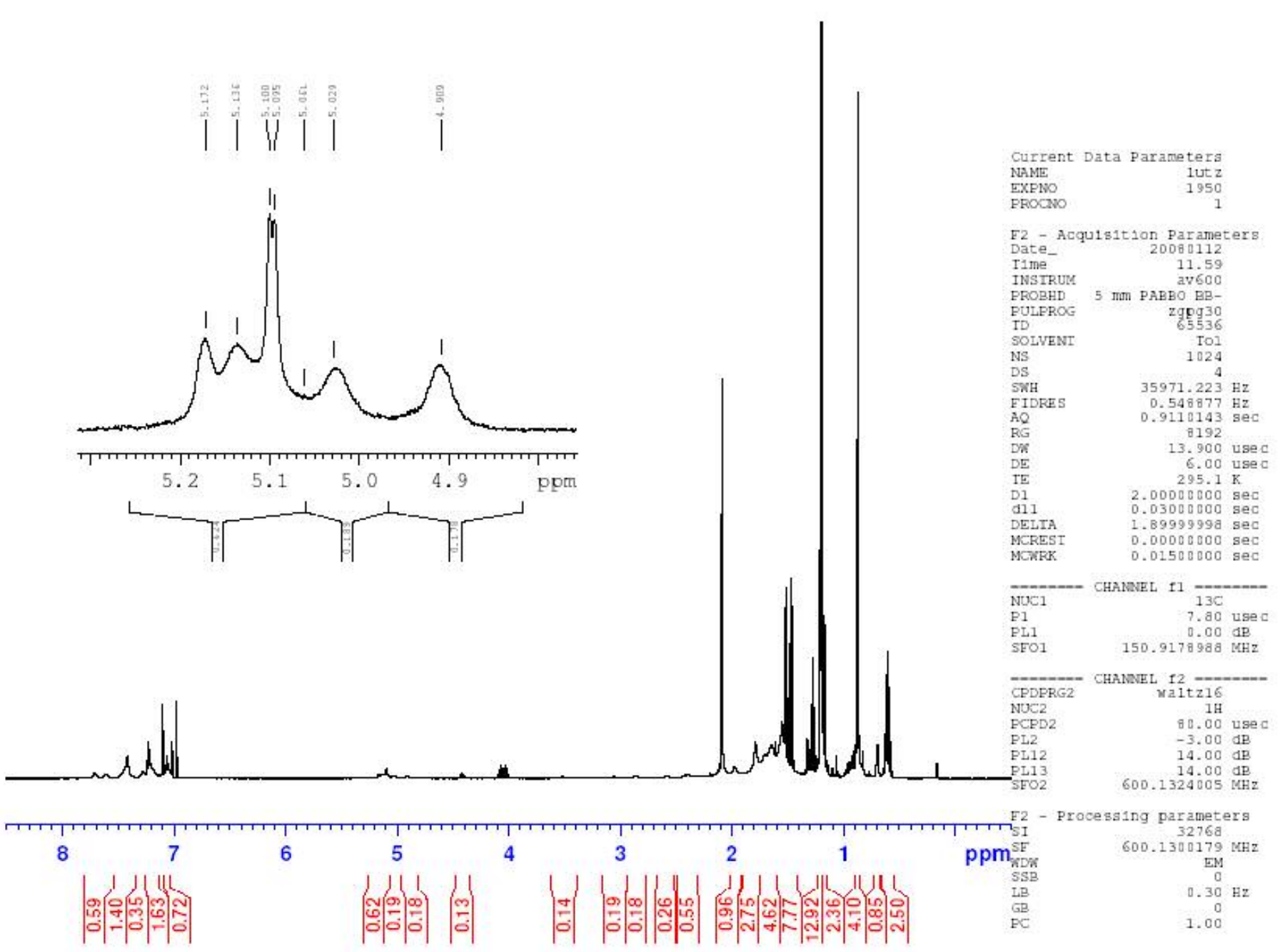


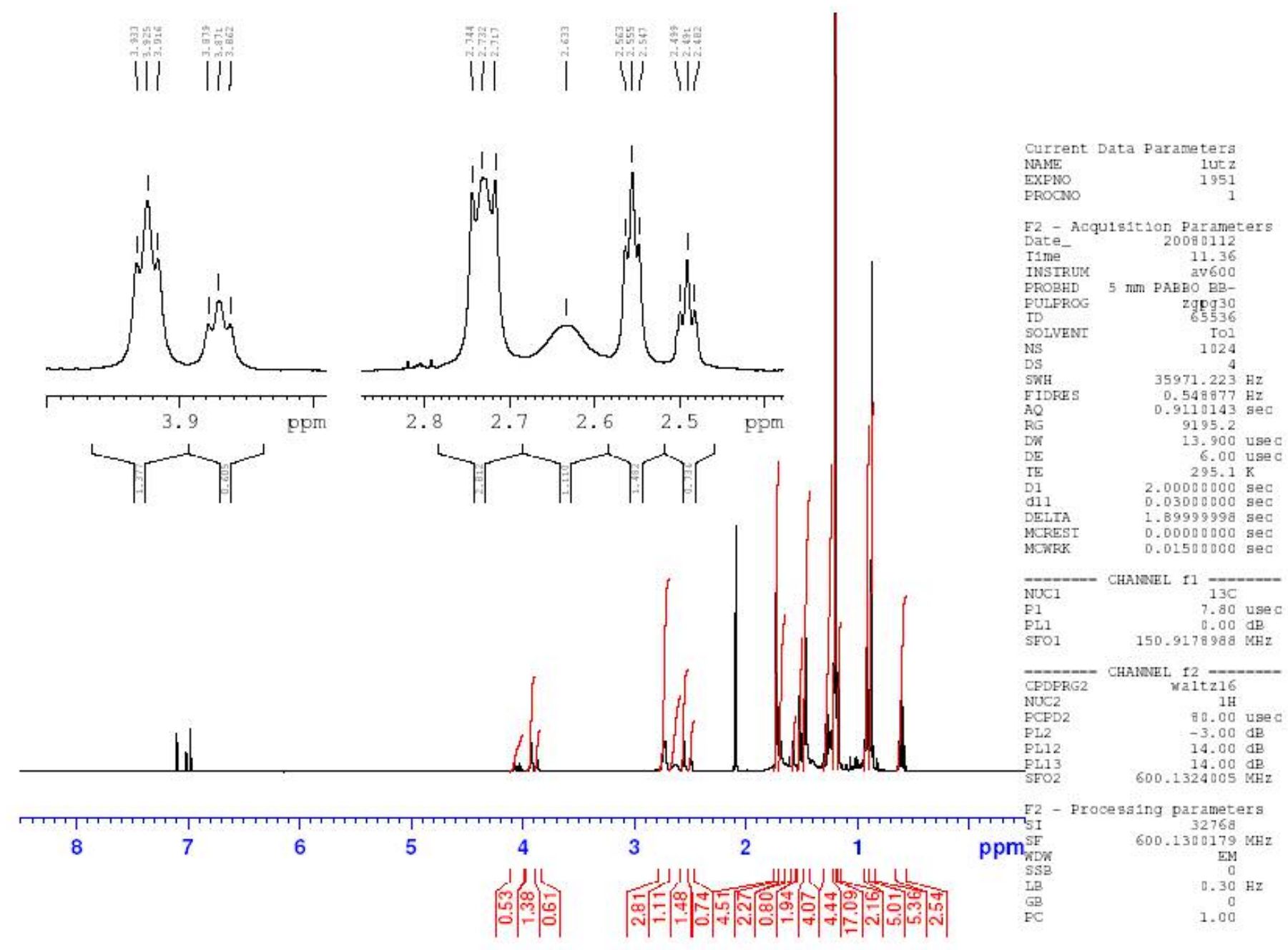




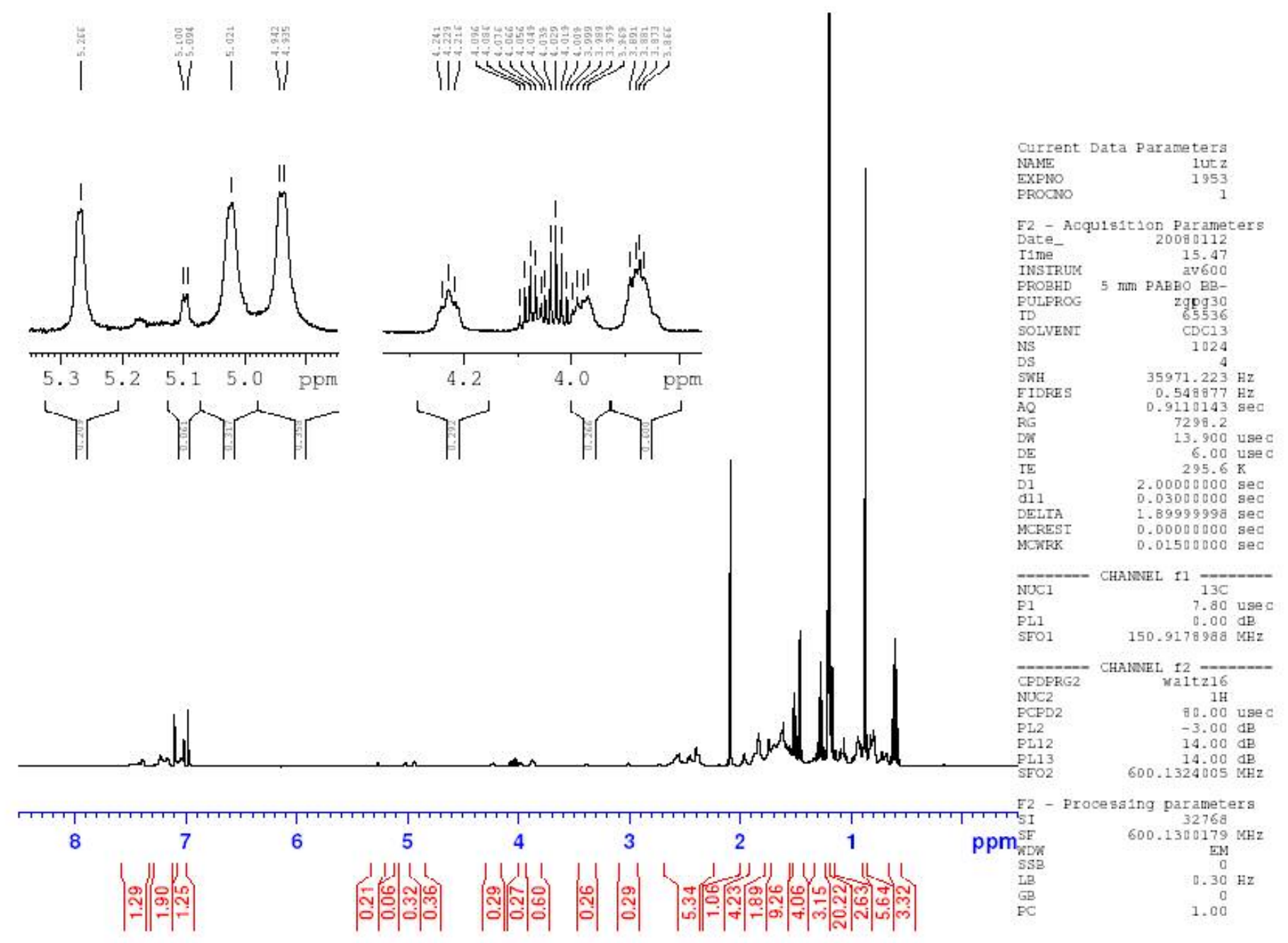




\section{Computational details:}

The ab initio calculations of the 5 possible monomeric and dimeric isopropylzinc alkoxides issued from DMNE and DMAE (shown in Scheme S-1) were carried out with Spartan 6.0 software (Wavefunction, Inc. 18401 Von Karman Avenue, Suite 370, Irvine, CA 92612 USA). For each structure a full optimization was performed at the restricted Hartree-Fock (RHF) level using energy gradient techniques, by using 3-21G basis set for $\mathrm{Zn}$ and 6-31G basis set for $\mathrm{C}, \mathrm{H}, \mathrm{N}$ and $\mathrm{O}$. The zero-point energies were determined at this level. The energy determination was performed by using B3LYP/6-31G* on the optimized geometries (B3LYP/LACVP* also gave similar estimations of enthalpies).

Scheme S-1. Calculated structures of monomeric and dimeric species issued from DMNE and DMAE

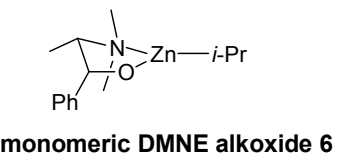

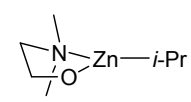

monomeric DMAE alkoxide 7

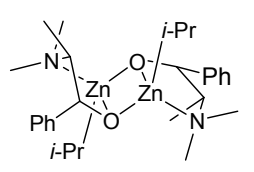

dimeric DMNE dialkoxide 8

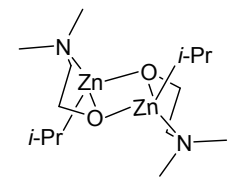

dimeric DMAE dialkoxide 9

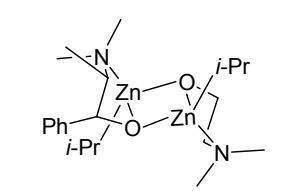

dimeric mixed dialkoxide 10

Table S-1. Total energies and zero-point energies (ZPE) of 6-10 (in Hartrees).

\begin{tabular}{|c|c|c|}
\hline Compound & $\begin{array}{c}\text { Total Energy } \\
(\text { B3LYP/6-31G*) }\end{array}$ & $\begin{array}{c}\text { Zero-Point Energy } \\
(\text { RHF/6-31G) }\end{array}$ \\
\hline $\mathbf{6}$ & -2186.09677 & 0.25458 \\
\hline $\mathbf{7}$ & -2456.46039 & 0.37193 \\
\hline $\mathbf{8}$ & -4912.97140 & 0.74653 \\
\hline $\mathbf{9}$ & -4372.25411 & 0.51134 \\
\hline $\mathbf{1 0}$ & -4642.61699 & 0.62867 \\
\hline
\end{tabular}


S-22 -

The optimized geometries are shown in Figure S-7 11 (red: oxygen; gray: carbon; green: zinc; blue: nitrogen; hydrogen atoms are not shown for clarity).

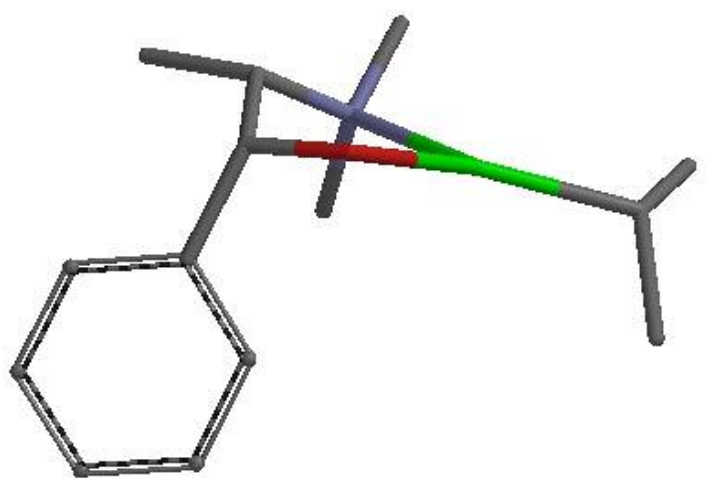

Figure S-7. Calculated structure of 6

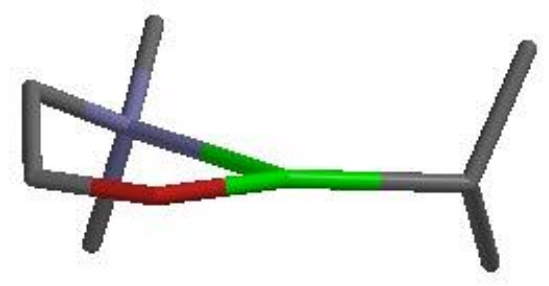

Figure S-8. Calculated structure of 7

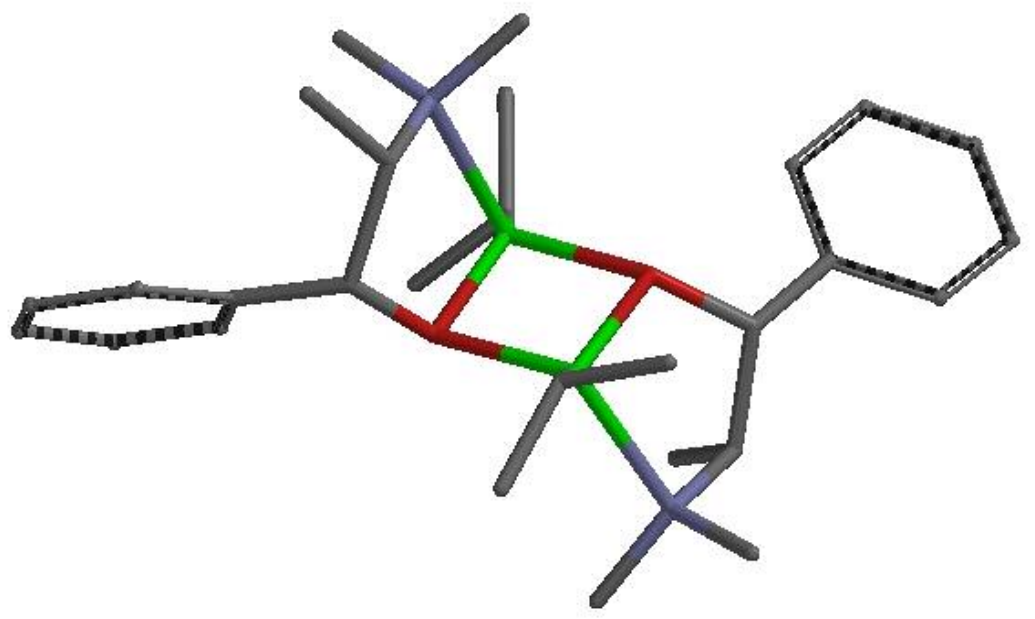

Figure S-9. Calculated structure of 8 


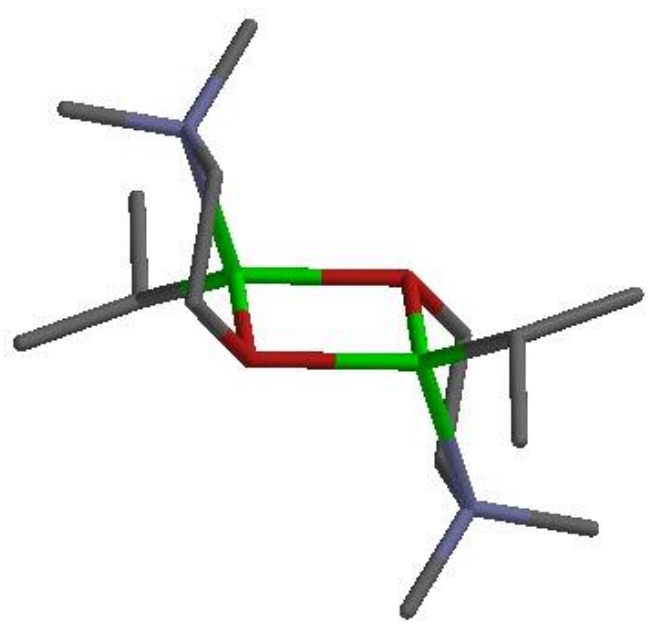

Figure S-10. Calculated structure of 9

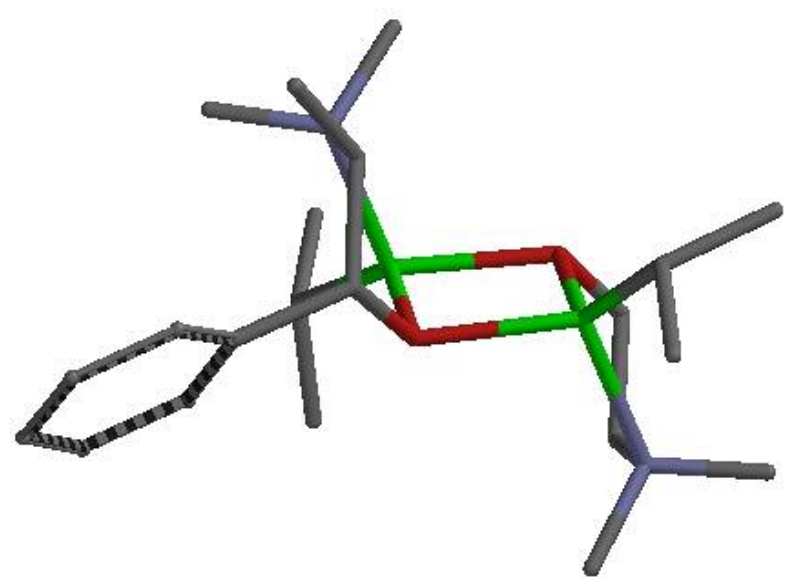

Figure S-11. Calculated structure of $\mathbf{1 0}$ 